\title{
Malária durante a gravidez: efeito sobre o curso da gestação na região amazônica ${ }^{1}$
}

\author{
Eda Cristina da Silva Chagas, ${ }^{2,3,4}$ Cristiana Teixeira do Nascimento, ${ }^{2}$ \\ Franklin Simões de Santana Filho, ${ }^{2,3,4}$ Camila Helena Bôtto-Menezes ${ }^{3}$ \\ e Flor Ernestina Martinez-Espinosa ${ }^{2,4,5}$
}

Como citar Chagas ECS, Nascimento CT, Santana Filho FS, Bôtto-Menezes CH, Martinez-Espinosa FE. Malária durante a gravidez: efeito sobre o curso da gestação na região amazônica. Rev Panam Salud Publica. 2009;26(3):203-08.

RESUMO Objetivo. Estimar o efeito da malária sobre o curso da gestação em mulheres na região amazônica e investigar possíveis fatores de risco nessa população.

Métodos. Este estudo transversal é parte de um projeto maior para estudar malária e gravidez na região amazônica. Foram incluídas gestantes com malária atendidas na Fundação de Medicina Tropical do Amazonas que responderam a entrevistas estruturadas. Dados socioeconômicos, comportamentais e clínicos foram levantados na primeira consulta relacionada a cada novo episódio de malária na gestante. Todas as gestantes foram acompanhadas ao longo de sua gestação. Foram considerados os seguintes fatores de risco para alterações no curso da gestação: idade materna menor do que 20 anos, primeira gestação, primeira infecção malárica e espécie de plasmódio infectante.

Resultados. Foram avaliados 535 episódios de malária em 417 gestantes, sendo 20,56\% causados pelo P. falciparum, 78,69\% pelo $\mathrm{P}$. vivax e 0,75\% pela associação dos dois parasitas. Alteração no curso da gestação foi um evento muito frequente (26,2\%). Ameaça de aborto ocorreu em 49 casos (25,5\%), aborto em dois $(1,0 \%)$, ameaça de parto prematuro em 74 $(25,1 \%)$ e parto prematuro em três $(1,0 \%)$. Ser primigesta e adolescente apresentou associação estatisticamente significativa com ameaça de parto prematuro e abortamento.

Conclusão. A alteração no curso da gestação foi um evento muito frequente durante o episódio agudo de malária, embora a interrupção da gestação tenha tido baixa ocorrência em nossa casuística. Os resultados não evidenciaram um fator de risco de destaque, sugerindo que qualquer gestante pode apresentar ameaça de interrupção ou interrupção da gestação na vigência de episódio agudo de malária.

Palavras-chave Malária; complicações parasitárias na gravidez; aborto; ameaça de aborto; trabalho de parto prematuro; parto prematuro; Plasmodium vivax; Plasmodium falciparum.

1 Este trabalho faz parte da dissertação de mestrado de Eda Cristina da Silva Chagas, apresentada ao Programa de Pós-Graduação em Doenças Tropicais e Infecciosas da Universidade do Estado do Amazonas em convênio com a Fundação de Medicina Tropical do Amazonas (UEA/FMTAM).

2 Fundação de Medicina Tropical do Amazonas, Manaus (AM), Brasil.

3 Universidade Federal do Amazonas, Manaus (AM), Brasil.

4 Centro Universitário Nilton Lins, Manaus (AM), Brasil. E-mail: eda-chagas@uol.com.br

5 Centro de Pesquisas Leônidas e Maria Deane/ Fundação Oswaldo Cruz. Correspondência: Cen-
Da concepção até o parto, uma enorme variedade de eventos pode aumentar a complexidade da gravidez, causando, algumas vezes, a sua interrupção antes do desfecho esperado (1). Alguns desses eventos sofrem variações conforme o con-

tro de Pesquisas Leônidas e Maria Deane, Rua Terezina 476, Bairro Adrianópolis, CEP 69057-170, Manaus, AM, Brasil. Fone: +55-92-3621.2323; fax: +55-92-3621.2399; e-mail: florespinosa@gmail.com texto epidemiológico, como no caso da malária.

Estima-se que, a cada ano, aproximadamente 50 milhões de mulheres residentes em países endêmicos para malária engravidem e enfrentem, consequentemente, um risco aumentado de adquirir malária e suas complicações. A infecção por plasmódios durante a gestação é responsável por 10000 óbitos maternos, um 
número não estimado de abortos e por aproximadamente 20000 óbitos em crianças no primeiro ano de vida, como consequência do baixo peso ao nascer e da morte perinatal (2).

No Brasil, a quase totalidade dos casos de malária ocorre na Amazônia Legal, onde permanecem condições ecologicamente propícias à transmissão, em função dos fenômenos ambientais que facilitam a manutenção e a formação de criadouros naturais do anofelino, o principal vetor da doença (3). Manaus é um dos três municípios (com Porto Velho e Cruzeiro do Sul) responsáveis por 22,5\% dos casos de malária notificados no Brasil. Uma incidência parasitária anual de 29 casos por 1000 habitantes qualifica Manaus como um município de médio risco de transmissão $(4,5)$.

Em gestantes, a real frequência da malária ainda é desconhecida, tanto no Brasil como em toda a região das Américas; porém, no futuro, essa cifra poderá ser estimada em função de estudos multicêntricos atualmente em andamento e, no caso particular do Brasil, em função da determinação do Ministério da Saúde de que a gota espessa tenha status de exame de rotina no controle prenatal de gestantes residentes em áreas endêmicas de malária. Em 2001 e 2002, um inquérito epidemiológico de base populacional realizado no Município de Coari (Estado do Amazonas) encontrou uma prevalência de infecção de 4,3\% em gestantes, contra uma prevalência de $0,8 \%$ em mulheres não gestantes da mesma faixa etária (6).

As mulheres grávidas são particularmente vulneráveis à malária. Isso ocorre porque a gravidez altera o estado de imunidade, tornando a mulher mais suscetível à infecção malárica e aumentando o risco de formas complicadas da doença, como anemia grave da malária e óbito materno. Para a criança, a malária materna aumenta o risco de aborto espontâneo, natimortalidade, prematuridade e baixo peso ao nascer, representando uma importante causa de mortalidade infantil (7).

O desenvolvimento de imunidade contra a malária é lento e está estreitamente ligado à frequência e à duração da exposição ao plasmódio. O efeito que a malária exerce sobre a mãe e o feto ou o recém-nascido está diretamente relacionado à endemicidade da região onde residem. A densidade e a prevalência da parasitemia estão aumentadas na mulher grávida se comparada com a não grávida que resida na mesma região geográfica. A maior suscetibilidade da grávida não imune parece estar relacionada à deficiência da imunidade celular. Além disso, o fato de a placenta se constituir num local propício para a multiplicação do parasita torna as grávidas mais susceptíveis à malária, com exacerbação dos sintomas e maior risco de complicações (8). A malária durante a gravidez predispõe a alterações na evolução da gestação, sendo responsável por vários efeitos indesejáveis tanto na gestante como no concepto, sendo causa frequente de ameaça de aborto, aborto, ameaça de parto prematuro, parto prematuro, baixo peso ao nascer, retardo do crescimento intrauterino e anemia materna (9).

A característica clínica da malária durante a gravidez varia de acordo com o grau de imunidade que a mulher adquiriu durante a vida até o início da gravidez e conforme sua situação epidemiológica. Poucas infecções em adultos saudáveis que vivem em áreas de alta transmissão de malária resultam em febre. O mesmo se aplica para mulheres grávidas semiimunes, sendo comum que a maioria das grávidas com parasitemia apresentem poucos sintomas (10).

Sendo assim, muito se sabe sobre a suscetibilidade aumentada das mulheres grávidas para infecção e doença de malária, inclusive na região amazônica (6, 11-13). Porém, como mencionamos anteriormente, a magnitude da doença nesse grupo, assim como a fisiopatologia da malária materna e as dificuldades específicas do tratamento só recentemente se tornaram foco de pesquisa. Portanto, são necessários estudos sobre o impacto da malária na gestação, que estimem a frequência das principais alterações no curso da gravidez e identifiquem os possíveis fatores associados com a infecção, a fim de subsidiar as políticas públicas de prevenção e assistência.

O objetivo do presente estudo foi estimar a frequência de alterações no curso da gravidez em gestantes com episódio agudo de malária, assim como identificar possíveis fatores de risco predisponentes a essas alterações nessa população.

\section{MATERIAIS E MÉTODOS}

Foram analisadas neste estudo as seguintes alterações da gestação: ameaça de aborto, aborto, ameaça de parto prematuro e parto prematuro em gestantes que apresentavam formas assexuadas de parasitas do gênero Plasmodium sp. no sangue periférico.

\section{Desenho do estudo}

Este estudo transversal, realizado em uma coorte de mulheres com diagnóstico de malária, faz parte do projeto "Malária \& gravidez na região amazônica: diagnóstico, tratamento e acompanhamento clínico-laboratorial de pacientes da Fundação de Medicina Tropical do Amazonas (FMTAM)", que estabeleceu em 2005 o ambulatório especial de malária e gravidez na FMTAM. Foi constituída uma coorte para observar eventos associados ao binômio malária-gravidez, cujas características serão descritas em outra publicação. Dados socioeconômicos, comportamentais e clínicos foram coletados a partir de entrevista estruturada na primeira consulta realizada a cada novo episódio de malária na gestante. Foram considerados como possíveis fatores de risco para alterações da gestação nessas pacientes a idade materna menor do que 20 anos (adolescência), primeira gestação, primeira infecção malárica e espécie de plasmódio infectante.

\section{População de estudo}

Foram estudadas gestantes com diagnóstico de malária que procuraram o ambulatório da FMTAM de forma espontânea para tratamento de malária no período de março de 2005 a março de 2007. Eram elegíveis para o estudo as mulheres com exame positivo de gota espessa e diagnóstico laboratorial, ultrassonográfico ou clínico de gravidez. Todas as participantes assinaram um termo de consentimento livre e esclarecido. O projeto foi aprovado pelo Comitê de Ética em Pesquisa da FMTAM, sob o número 0836-05.

Para efeitos do presente estudo, foram consideradas como alteração no curso da gestação a ameaça de interrupção ou a interrupção da gestação em mulheres com infecção por formas assexuadas de plasmódio no sangue periférico. Definiuse como ameaça de interrupção da gravidez a presença de sinais ou sintomas tais como contrações uterinas, sangramento uterino ou amniorrexe. A interrupção da gestação foi definida como a expulsão espontânea total ou parcial do conteúdo uterino. Dessa forma, quatro possíveis desfechos foram observados: ameaça de aborto ou ameaça de interrupção da ges- 
tação antes de 22 semanas de idade gestacional; aborto ou interrupção da gravidez por expulsão de embrião/feto antes de 22 semanas de gestação ou com peso inferior a 500 gramas; ameaça de parto prematuro ou ameaça de interrupção da gestação com idade gestacional entre 22 e 37 semanas incompletas; e parto prematuro quando houve a interrupção da gestação (10).

\section{Definições}

A idade gestacional foi definida como o tempo de amenorreia em semanas a contar do início da última menstruação e/ou corrigido por ultrassom realizado nas primeiras semanas de gravidez. O episódio agudo de malária foi definido como o período transcorrido entre o início dos sintomas e a primeira lâmina negativa de controle, o que, na maioria dos casos, coincidiu com o terceiro dia de seguimento. Definiu-se primoinfecção como o primeiro episódio clínico de malária durante toda a vida do paciente. Morte fetal foi definida como a morte antes da expulsão ou extração completa do produto de concepção, independentemente da duração da gravidez, sendo indicada pela ausência de evidência de vida fetal. A morte materna correspondeu à morte da mulher enquanto grávida ou dentro de 42 dias após o término da gravidez. O período neonatal foi considerado como aquele que começa com o nascimento e termina 28 dias após o parto. A morte neonatal compreendeu a morte do concepto durante os primeiros 28 dias de vida, sendo precoce quando ocorreu até o $7^{\circ}$ dia e tardia após o $7^{\circ}$ dia de vida. Foram consideradas prematuras as crianças nascidas com menos de 37 semanas de gestação (gestação com duração de menos de 259 dias). A adolescência foi definida na faixa etária dos 10 aos 19 anos, correspondendo ao período de amadurecimento físico, psicológico e social (10).

\section{Análise estatística}

Os dados foram armazenados e analisados no programa Epi-Info 6.04. Foram calculados as médias e os desviospadrão para variáveis quantitativas, e a distribuição de frequência para variáveis qualitativas. Para estabelecer diferenças estatisticamente significativas entre as médias foi utilizado o teste $t$ de Student com correção de Yates ou de Fisher, se- gundo o caso. A diferença entre proporções foi mensurada com o teste do qui-quadrado $\left(\chi^{2}\right)$, utilizando-se um intervalo de confiança de 95\% (IC95\%) e uma significância estatística de $P<0,05$ para descartar a hipótese nula. Para estabelecer a associação entre variáveis primárias e secundárias foi estimada a razão de chances (odds ratio, OR), utilizando-se o mesmo IC95\% e $P<0,05$ acima citados.

\section{RESULTADOS}

Durante o período do estudo, foram identificados 535 episódios de malária em 417 gestantes. Essas 417 gestantes, no momento do episódio em avaliação, tinham idade entre 9,5 e 44,6 anos e possuíam baixa escolaridade (média de $6,3 \pm 3,2$ anos completos de estudo). Eram predominantemente casadas ou com vida conjugal estável $(79,7 \%)$. A ocupação de dona de casa foi referida por $64,4 \%$. A média de paridade foi significativamente maior entre as pacientes que apresentaram alterações no curso da gestação (tabela 1).

Dos 535 episódios de malária nessas gestantes, $110(20,56 \%)$ foram causados pelo $P$. falciparum, $421(78,69 \%)$ pelo $P$. vivax e cinco $(0,75 \%)$ pela associação dos dois parasitas (malária mista). A primoinfecção foi encontrada em 109 (20,84\%) episódios. Não houve associação estatisticamente significativa entre a espécie causadora de infecção ou de primoinfecção e a ocorrência de alterações na evolução da gestação (tabela 2); porém, a média de paridade foi maior entre as gestantes com alteração no curso de gestação, sendo essa diferença estatisticamente significativa (tabela 1).

\section{Desfecho da gestação}

No momento do episódio, 192 (35,88\%) gestantes tinham menos de 22 semanas de amenorreia, estando, portanto, em risco de aborto; 295 (55,14\%) estavam entre 22 e menos de 37 semanas, em risco de parto prematuro, e $48(8,97 \%)$ tinham gravidez maior ou igual a 37 semanas. De um modo geral, a alteração na evolução da gestação foi um evento frequente $(26,2 \%)$, sendo que a ameaça de aborto ocorreu em 49 $(25,5 \%)$ das gestantes em risco, o aborto em duas $(1,0 \%)$, a ameaça de parto prematuro ocorreu em $74(25,1 \%)$ e o parto prematuro em três $(1,0 \%)$ das gestantes em risco.

\section{Associação entre fatores de risco e desfecho da gestação}

A tabela 3 mostra os dados relativos à associação entre possíveis fatores de risco, ameaça de parto prematuro e abortamento durante episódio agudo de malária. Ser primigesta e adolescente apresentou associação estatisticamente significativa com a ameaça de parto prematuro. A adolescência esteve associada a abortamento entre as gestantes com malária.

\section{DISCUSSÃO}

A alteração no curso da gestação, avaliada neste estudo por quatro eventos possíveis, mostrou ser muito frequente durante o episódio agudo de malária, embora a interrupção da gestação durante a infecção aguda de malária tenha sido de baixa frequência. Isto pode ser atribuído ao fato de a casuística aqui apresentada ter sido composta de pacientes que tiveram como forma de apresentação clínica os sintomas gerais de malária, motivando a procura por diagnóstico de malária em centro de referência para doenças infecciosas e, portanto, não contabilizando as grávidas com malária que apresentem sintomatologia obstétrica inicialmente e que, em consequência, procuram atendimento em serviços obstétricos.

Adicionalmente, a baixa frequencia de interrupção da gestação aqui observada
TABELA 1. Alterações no curso da gestação em mulheres com malária atendidas na Fundação de Medicina Tropical do Amazonas, Brasil, 2005 a 2007

\begin{tabular}{lccc}
\hline & \multicolumn{3}{c}{ Alterações no curso da gestação } \\
\cline { 2 - 4 } \multicolumn{1}{c}{ Variável (média \pm DP) } & Sim & Não & \\
\hline Idade materna (anos) & $140(26,2 \%)^{\mathrm{a}}$ & $395(73,8 \%)^{\mathrm{a}}$ & $P$ \\
Idade gestacional (semanas) & $23,95 \pm 5,72$ & $23,87 \pm 5,99$ & 0,89 \\
Paridade (gestações) & $25,12 \pm 8,54$ & $24,96 \pm 8,76$ & 0,92 \\
& $3,01 \pm 2,19$ & $2,59 \pm 1,87$ & 0,03 \\
\hline
\end{tabular}

a Em relação ao total de episódios ocorridos em 417 pacientes, $n=535$. 
TABELA 2. Associação entre possíveis fatores de risco e sintomas compatíveis com alteração do curso da gestação em mulheres com malária atendidas na Fundação de Medicina Tropical do Amazonas, Brasil, 2005 a 2007

\begin{tabular}{|c|c|c|c|c|c|c|c|}
\hline \multirow[b]{3}{*}{ Fatores de risco } & \multicolumn{7}{|c|}{ Alterações no curso da gestação } \\
\hline & \multicolumn{2}{|r|}{ Sim } & \multicolumn{2}{|r|}{ Não } & OR & $\mathrm{IC} 95 \%$ & $P$ \\
\hline & \multicolumn{7}{|c|}{ Contrações uterinas } \\
\hline Primigestação & 23 & $(13,5 \%)$ & 147 & $(86,5 \%)$ & 0,8 & 0,47 a 1,35 & 0,45 \\
\hline Primoinfecção & 20 & $(18,3 \%)$ & 89 & $(81,7 \%)$ & 0,87 & 0,49 a 1,53 & 0,70 \\
\hline Adolescência & 22 & $(15,2 \%)$ & 123 & $(84,2 \%)$ & 0,65 & 0,38 a 1,12 & 0,12 \\
\hline \multicolumn{8}{|l|}{ Espécie infectante } \\
\hline Plasmodium vivax & 84 & $(20,4 \%)$ & 327 & $(79,6 \%)$ & 1,22 & 0,68 a 2,19 & 0,57 \\
\hline Plasmodium falciparum & 19 & $(17,4 \%)$ & 90 & $(82,6 \%)$ & & & \\
\hline Febre & 95 & $(21,5 \%)$ & 346 & $(78,5 \%)$ & 2,07 & 1,02 a 4,29 & $0,04^{\mathrm{a}}$ \\
\hline \multicolumn{8}{|l|}{ Amenorreia } \\
\hline$<22$ semanas & 34 & $(17,8 \%)$ & 157 & $(82,2 \%)$ & 0,82 & 0,51 a 1,32 & 0,44 \\
\hline \multirow[t]{2}{*}{$\geq 22$ a $<37$ semanas } & 72 & $(20,9 \%)$ & 272 & $(79,1 \%)$ & & & \\
\hline & \multicolumn{7}{|c|}{ Sangramento vaginal } \\
\hline Primigestação & 11 & $(6,5 \%)$ & 159 & $(93,5 \%)$ & 1,76 & 0,73 a 4,24 & 0,24 \\
\hline Primoinfecção & 9 & $(36,0 \%)$ & 16 & $(64,0 \%)$ & 2,26 & 0,89 a 5,62 & 0,09 \\
\hline Adolescência & 12 & $(8,3 \%)$ & 133 & $(91,7 \%)$ & 2,62 & 1,09 a 6,28 & $0,03^{a}$ \\
\hline \multicolumn{8}{|l|}{ Espécie infectante } \\
\hline Plasmodium vivax & 20 & $(4,9 \%)$ & 391 & $(95,1 \%)$ & 1,06 & 0,37 a 3,32 & 0,89 \\
\hline Plasmodium falciparum & 5 & $(4,6 \%)$ & 104 & $(95,4 \%)$ & & & \\
\hline Febre & 22 & $(5,0 \%)$ & 419 & $(95,0 \%)$ & 1,59 & 0,44 a 6,83 & 0,63 \\
\hline \multicolumn{8}{|l|}{ Amenorreia } \\
\hline$<22$ semanas & 12 & $(6,3 \%)$ & 179 & $(93,7 \%)$ & 1,71 & 0,71 a 4,08 & 0,27 \\
\hline \multirow[t]{2}{*}{$\geq 22$ a $<37$ semanas } & 13 & $(3,8 \%)$ & 331 & $(96,2 \%)$ & & & \\
\hline & \multicolumn{7}{|c|}{ Amniorrexe } \\
\hline Primigestação & 6 & $(3,5 \%)$ & 164 & $(96,5 \%)$ & 1,09 & 0,38 a 3,01 & 0,95 \\
\hline Primoinfecção & 3 & $(2,8 \%)$ & 106 & $(97,2 \%)$ & 0,56 & 0,13 a 2,05 & 0,50 \\
\hline Adolescência & 6 & $(4,1 \%)$ & 139 & $(95,9 \%)$ & 0,95 & 0,33 a 2,62 & 0,89 \\
\hline \multicolumn{8}{|l|}{ Espécie infectante } \\
\hline Plasmodium vivax & 19 & $(4,6 \%)$ & 392 & $(95,4 \%)$ & 1,27 & 0,4 a 4,52 & 0,86 \\
\hline Plasmodium falciparum & 4 & $(3,7 \%)$ & 105 & $(96,3 \%)$ & & & \\
\hline Febre & 20 & $(4,5 \%)$ & 421 & $(95,5 \%)$ & 1,44 & 0,39 a 6,23 & 0,76 \\
\hline \multicolumn{8}{|l|}{ Amenorreia } \\
\hline$<22$ semanas & 6 & $(3,1 \%)$ & 185 & $(96,9 \%)$ & 0,62 & 0,22 a 1,72 & 0,44 \\
\hline$\geq 22$ a $<37$ semanas & 17 & $(4,9 \%)$ & 327 & $(95,1 \%)$ & & & \\
\hline
\end{tabular}

${ }^{a}$ Correção de Yates.

pode ser atribuída ao diagnóstico e à intervenção precoces, impedindo, com isso, a evolução natural da doença e preservando a saúde materno-infantil. Conhecer o tamanho do fardo da malária em relação ao desfecho da gestação será possível quando, de modo rotineiro, se procure a causa etiológica de todos os abortos "espontâneos" e partos prematuros com ou sem perda do concepto que ocorrem nas áreas endêmicas.

A malária na gestação é potencialmente grave, sendo causa indireta de morbidade e mortalidade perinatal, especialmente no terceiro trimestre de gestação, com evidência de menor ocorrência nos primeiros trimestres. Dados recentes sugerem que as gestantes que residem em áreas endêmicas, que apresentam infecção placentária, principalmente as primigestas, apresentam risco aumentado de anemia e alteração na circulação útero-placentária, determinando deficiência de nutrientes, contribuindo para o baixo peso ao nascimento (por prematuridade ou retardo no crescimento intrauterino) e mortalidade infantil (14). Estima-se que a malária materna contribua com taxa de $3 \%$ para a mortalidade infantil, sem considerar as alterações decorrentes da anemia materna na mortalidade infantil (15).

As características clínicas dependem do estado imune da mulher, determinado nos últimos anos de exposição ao plasmódio. Pacientes grávidas não imunes são mais propensas a adquirir doença grave do que mulheres não grávidas. Mulheres grávidas de qualquer paridade tem 1 a 2 vezes mais risco de desenvolver doença grave quando comparadas com mulheres não grávidas, e aproximadamente 3 vezes mais risco de morte materna em caso de doença grave. A malária grave em gestantes está associada a 20 a $30 \%$ de morte materna, enquanto que a mortalidade perinatal está relacionada à febre alta materna, causando contrações uterinas (15).

A contribuição da malária na gravidez para a mortalidade infantil não foi estimada na nossa região, mas a ampla distribuição geográfica do plasmódio ao redor dos trópicos torna a malária gestacional um importante problema de saúde pública. Contudo, vale ressaltar que as mulheres constituem um grupo que costumeiramente busca cuidado médico ativamente, principalmente durante a gestação, representando uma oportunidade de intervenção através do diagnóstico precoce e do tratamento adequado, com acompanhamento durante toda a gestação, reduzindo, assim, o prejuízo para a saúde materno-fetal e contribuindo para a redução da mortalidade infantil, sobretudo nos primeiros anos de vida. Outro fator que merece destaque é a tendência a subestimar os efeitos deletérios da malária na gestação e os reduzidos estudos sobre internações em unidades pediátricas de tratamento intensivo devido a parto prematuro e baixo peso ao nascimento decorrente de episódio de malária materna, que motivou o parto prematuro.

As gestantes que residem em áreas de transmissão estável de malária adquirem imunidade protetora na sua maturidade sexual, havendo indícios de importante associação de morbidez e mortalidade relacionada à malária, principalmente em primíparas $(16,17)$. Contudo, nas gestantes aqui estudadas, observou-se a ocorrência de alterações prejudiciais ao desenvolvimento da gestação principalmente no grupo de multíparas, sugerindo que essa suscetibilidade aparentemente independente da paridade seja decorrente de imunidade protetora desenvolvida mediante sucessivas exposições, dificultando, dessa forma, a determinação dos fatores de risco para a gestação. Portanto, ser multigesta parece não oferecer proteção contra os riscos impostos pela malária na gestação na região estudada.

O grupo de gestantes analisadas apresentou grande variação na faixa etária, verificando-se que as alterações no curso da gravidez e as consequentes repercussões na saúde materno-fetal ocorreram predominantemente nas mulheres na faixa etária jovem, no segundo tri- 
TABELA 3. Associação entre possíveis fatores de risco e alterações no curso da gestação durante episódio agudo de malária em mulheres atendidas na Fundação de Medicina Tropical do Amazonas, Brasil, 2005 a 2007

\begin{tabular}{|c|c|c|c|c|c|c|}
\hline \multirow[b]{3}{*}{ Fatores de risco } & \multicolumn{6}{|c|}{ Alterações no curso da gestação } \\
\hline & Sim & & Não & OR & IC95\% & $P$ \\
\hline & \multicolumn{6}{|c|}{ Ameaça de aborto } \\
\hline Primigestação & $17(26,6 \%)$ & 47 & $(73,4 \%)$ & 1,25 & 0,58 a 2,65 & $0,66^{a}$ \\
\hline Primoinfecção & $12(23,5 \%)$ & 39 & $(73,5 \%)$ & 1,01 & 0,44 a 2,29 & $>0,5$ \\
\hline \multirow[t]{2}{*}{ Adolescência } & $17(30,4 \%)$ & 39 & $(69,6 \%)$ & 2,39 & 1,12 a 5,1 & $0,02^{a}$ \\
\hline & \multicolumn{6}{|c|}{ Aborto } \\
\hline Primigestação & $1 \quad(2,8 \%)$ & 35 & $(97,2 \%)$ & 1,66 & 0,0 a 63,1 & $0,7^{b}$ \\
\hline Primoinfecção & $1 \quad(3,4 \%)$ & 28 & $(96,6 \%)$ & 2,25 & 0,0 a 86,01 & $0,5^{\mathrm{b}}$ \\
\hline \multirow[t]{2}{*}{ Adolescência } & $1 \quad(3,6 \%)$ & 27 & $(96,4 \%)$ & 1,48 & 0,0 a 57,16 & $0,64^{b}$ \\
\hline & \multicolumn{6}{|c|}{ Ameaça de parto prematuro } \\
\hline Primigestação & $16(16,2 \%)$ & 83 & $(83,8 \%)$ & 0,47 & 0,24 a 0,91 & $0,02^{a}$ \\
\hline Primoinfecção & $10(19,6 \%)$ & 41 & $(80,4 \%)$ & 1,01 & 0,45 a 2,24 & 0,087 \\
\hline \multirow[t]{2}{*}{ Adolescência } & $14(16,9 \%)$ & 69 & $(83,1 \%)$ & 0,54 & 0,27 a 1,07 & $0,05^{a}$ \\
\hline & \multicolumn{6}{|c|}{ Parto prematuro } \\
\hline Primigestação & $1 \quad(1,0 \%)$ & 98 & $(99,0 \%)$ & 0,97 & Inválido & 0,55 \\
\hline Primoinfecção & $1 \quad(2,0 \%)$ & 50 & $(98,0 \%)$ & 1,59 & Inválido & 0,79 \\
\hline Adolescência & $0 \quad(0,0 \%)$ & 83 & $(100,0 \%)$ & & & \\
\hline
\end{tabular}

a Correção de Yates.

${ }^{\mathrm{b}}$ Correção de Fisher.

mestre gestacional e em multigestantes. Isso sugere que a idade materna é um fator de risco adicional. Estudos têm sido realizados buscando correlacionar alterações no curso da gestação com possíveis fatores de risco, sendo apontada a idade materna como fator que agrava a doença, tendo em vista que o número de adolescentes com malária é proporcionalmente maior entre gestantes do que entre não gestantes, mostrando um potencial fator de risco adicional (11). Na região amazônica, um estudo evidenciou que $30 \%$ das gestantes hospitalizadas devido à malária eram menores de 20 anos de idade (18). Outro estudo realizado em Coari, no Amazonas, relatou que a ocorrência de malária entre adolescentes não diferiu da prevalência encontrada entre mulheres de outra faixa etária; con- tudo, quando a gravidez foi analisada como fator de risco para malária, a associação foi maior entre o grupo de menor idade (6).

\section{Conclusões}

A alteração no curso da gestação foi muito frequente em gestantes durante o episódio agudo de malária, sendo muito mais frequente a ameaça de interrupção do que a interrupção da gestação, cuja ocorrência foi baixa. Embora alguns antecedentes, tais como adolescência e apresentar febre ou multiparidade, estejam associados às alterações no curso da gestação, de forma geral os resultados mostraram que não houve fatores de risco identificáveis dentre aqueles estudados, tornando a gestante homogeneamente suscetível. O risco de apresentar alterações no curso da gestação na vigência de um episódio de malária parece ser semelhante independentemente da idade, paridade ou antecedentes de malária. Portanto, pode-se considerar que todas as gestantes devem ser alvo das ações de prevenção e controle da malária.

Agradecimentos. Este estudo foi financiado com recursos da Fundação de Amparo à Pesquisa do Amazonas (FAPEAM) /CNPq através do Programa de Desenvolvimento Científico Regional (DCR) outorgado a Flor Ernestina Martinez-Espinosa.

\section{REFERÊNCIAS}

1. Savitz DA, Hertz-Picciotto I, Poole C, Olshan AF. Epidemiologic measures of the course and outcome of pregnancy. Epidemiol Rev. 2002;24(2):91-101.

2. World Health Organization. The World Health Report 2005: make every mother and child count. Genebra: WHO; 2005. Disponível em: http://www.who.int/whr/2005/ en/index.html. Acessado em agosto de 2009.

3. Confalonieri UEC. Saúde na Amazônia: um modelo conceitual para a análise de paisagens e doenças. Estud Av. 2005;53(19): 221-36.

4. SIVEP-Malária. Notificação de casos. Relatório. Brasília: Secretária de Vigilância em Saúde/Sistema de Informações de Vigilância em Saúde/Ministério de Saúde; 2006.

5. Brasil, Ministério da Saúde. Situação epidemiológica de malária no Brasil 2007. Brasília: Secretaria de Vigilância em Saúde; 2007.
6. Martínez-Espinosa FE. Malaria e gravidez na região Amazônica: prevalência de infecção em mulheres de idade do Município de Coari, 2001-2002 [tese de doutorado]. Rio de Janeiro: Instituto Oswaldo Cruz - IOC/FIOCRUZ; 2003.

7. Alves M, Lima V, Rangel O. Malária congênita no município de Leme, SP. J Pediatr (Rio J). 1995;71(3):163-5.

8. Okoko BJ, Enwere G, Ota MO. The epidemiology and consequences of maternal malaria: a review of immunological basis. Acta Trop. 2003;87(2):193-205.

9. Menendez C. Malaria during pregnancy: a priority area of malaria research and control. Parasitol Today. 1995;11(5):178-82.

10. Desai M, ter Kuile FO, Nosten F, McGready R, Asamoa K, Brabin B, et al. Epidemiology and burden of malaria in pregnancy. Lancet Infect Dis. 2007;7(12):763-4.
11. Martínez-Espinosa FE. Malaria na gravidez: estudo de pacientes do Instituto de Medicina Tropical do Amazonas, Brasil, 1990-1997 [dissertação de mestrado]. Rio de Janeiro: Fundação Instituto Oswaldo Cruz; 1998.

12. Martinez-Espinosa FE, Alecrim WD, DanielRibeiro CT. Attraction of mosquitoes to pregnant women. Lancet. 2000;19;356(9230):685.

13. Martinez-Espinosa FE, Daniel-Ribeiro CT, Alecrim WD. Malaria during pregnancy in a reference centre from the Brazilian Amazon: unexpected increase of Plasmodium falciparum infections. Mem Inst Oswaldo Cruz. 2004; 99(1):19-21.

14. Steketee RW, Nahlen BL, Parise ME, Menen$\operatorname{dez} \mathrm{C}$. The burden of malaria in pregnancy in malaria-endemic areas. Am J Trop Med Hyg. 2001;64(1,2 Suppl):28-35.

15. Steketee RW, Wirima JJ, Campbell CC. Developing effective strategies for malaria preven- 
tion programs for pregnant African women. Am J Trop Med Hyg. 1996;55(1 Suppl):95-100.

16. Hviid L. The immuno-epidemiology of pregnancy-associated Plasmodium falciparum malaria: a variant surface antigenspecific perspective. Parasite Immunol. 2004; 26(11-12):477-86.
17. Brabin BJ, Ginny M, Sapau J, Galme K, Paino $\mathrm{J}$. Consequences of maternal anaemia on outcome of pregnancy in a malaria endemic area in Papua New Guinea. Ann Trop Med Parasit. 1990;84(1):11-24.

18. Jarude R, Trindade R, Tavares-Neto J. Malária em grávidas de uma maternidade pública de
Rio Branco (Acre, Brasil). RBGO. 2003;25(3): 149-54.

Manuscrito recebido em 27 de novembro de 2008. Aceito em versão revisada em 17 de março de 2009.

ABSTRACT Objective. To estimate the impact of malaria on the course of pregnancy in women from the Amazon region and to investigate possible risk factors in this population.

\section{Impact of malaria during pregnancy in the Amazon region}

Key words
Methods. This cross-sectional study is part of a larger project to study malaria and pregnancy in the Amazon region. Pregnant women with malaria receiving care at the Amazon Tropical Medicine Foundation (Fundação de Medicina Tropical do Amazonas) who answered a structured interview were included in the study. Socioeconomic, behavioral and clinical data were collected in the first consultation relating to each new malaria attack. All the women were followed-up throughout their pregnancy. The following risk factors for impact of malaria on the course of pregnancy were considered: being younger than 20 years of age, first pregnancy, first malaria infection, and type of infecting plasmodium species.

Results. Five hundred and thirty-five malaria episodes were evaluated in 417 pregnant women, with $20.56 \%$ being caused by P. falciparum, $78.69 \%$ by $P$. vivax and $0.75 \%$ by the association of both parasites. Changes in the course of pregnancy were very frequent $(26.2 \%)$. Threat of abortion was observed in 49 cases $(25.5 \%)$, abortion in two $(1.0 \%)$, threat of premature birth in $74(25.1 \%)$, and premature birth in three $(1.0 \%)$. First pregnancy and age $<20$ years were significantly associated with threat of premature birth and abortion.

Conclusion. Changes in the course of pregnancy were very frequent during acute malaria attacks, despite the low frequency of abortion. The present results do not highlight specific risk factors, suggesting that any pregnant woman may be at risk for the threat of abortion or for abortion during acute malaria attacks.

Malaria; pregnancy complications, parasitic; abortion; abortion, threatened; obstetric labor; premature; Plasmodium vivax; Plasmodium falciparum. 